\title{
Characteristics of the Diffuse Astrophysical Electron and Tau Neutrino Flux with Six Years of IceCube High Energy Cascade Data
}

M. G. Aartsen, ${ }^{16}$ M. Ackermann, ${ }^{55}$ J. Adams, ${ }^{16}$ J. A. Aguilar, ${ }^{12}$ M. Ahlers, ${ }^{20}$ M. Ahrens, ${ }^{46}$ C. Alispach, ${ }^{26}$ K. Andeen, ${ }^{37}$ T. Anderson, ${ }^{52}$ I. Ansseau, ${ }^{12}$ G. Anton, ${ }^{24}$ C. Argüelles, ${ }^{14}$ J. Auffenberg, ${ }^{1}$ S. Axani, ${ }^{14}$ P. Backes, ${ }^{1}$ H. Bagherpour, ${ }^{16}$ X. Bai, ${ }^{43}$ A. Balagopal V. ${ }^{29}$ A. Barbano, ${ }^{26}$ S. W. Barwick, ${ }^{28}$ B. Bastian, ${ }^{55}$ V. Baum, ${ }^{36}$ S. Baur, ${ }^{12}$ R. Bay, ${ }^{8}$ J. J. Beatty, ${ }^{18,19}$ K.-H. Becker,${ }^{54}$ J. Becker Tjus, ${ }^{11}$ S. BenZvi, ${ }^{45}$ D. Berley, ${ }^{17}$ E. Bernardini, ${ }^{55, \dagger}$ D. Z. Besson, ${ }^{30, \$}$ G. Binder, ${ }^{8,9}$ D. Bindig, ${ }^{54}$ E. Blaufuss, ${ }^{17}$ S. Blot,${ }^{55}$ C. Bohm, ${ }^{46}$ S. Böser,${ }^{36}$ O. Botner, ${ }^{53}$ J. Böttcher, ${ }^{1}$ E. Bourbeau, ${ }^{20}$ J. Bourbeau, ${ }^{35}$ F. Bradascio, ${ }^{55}$ J. Braun, ${ }^{35}$ S. Bron, ${ }^{26}$ J. Brostean-Kaiser, ${ }^{55}$ A. Burgman, ${ }^{53}$ J. Buscher, ${ }^{1}$ R. S. Busse ${ }^{38}$ T. Carver, ${ }^{26}$ C. Chen, ${ }^{6}$ E. Cheung, ${ }^{17}$ D. Chirkin, ${ }^{35}$ S. Choi ${ }^{48}$ K. Clark, ${ }^{31}$ L. Classen, ${ }^{38}$ A. Coleman, ${ }^{39}$ G. H. Collin, ${ }^{14}$ J. M. Conrad,${ }^{14}$ P. Coppin ${ }^{13}$ P. Correa, ${ }^{13}$ D. F. Cowen ${ }^{51,52}$ R. Cross,${ }^{45}$ P. Dave, ${ }^{6}$ C. De Clercq, ${ }^{13}$ J. J. DeLaunay, ${ }^{52}$ H. Dembinski, ${ }^{39}$ K. Deoskar, ${ }^{46}$ S. De Ridder, ${ }^{27}$ P. Desiati, ${ }^{35}$ K. D. de Vries, ${ }^{13}$ G. de Wasseige, ${ }^{13}$ M. de With, ${ }^{10}$ T. DeYoung, ${ }^{22}$ A. Diaz, ${ }^{14}$ J. C. Díaz-Vélez,${ }^{35}$ H. Dujmovic, ${ }^{29}$ M. Dunkman, ${ }^{52}$ E. Dvorak ${ }^{43}$ B. Eberhardt, ${ }^{35}$ T. Ehrhardt, ${ }^{36}$ P. Eller, ${ }^{52}$ R. Engel, ${ }^{29}$ P. A. Evenson,${ }^{39}$ S. Fahey, ${ }^{35}$ A. R. Fazely, ${ }^{7}$ J. Felde,${ }^{17}$ K. Filimonov,${ }^{8}$ C. Finley, ${ }^{46}$ D. Fox ${ }^{51}$ A. Franckowiak, ${ }^{55}$ E. Friedman, ${ }^{17}$ A. Fritz, ${ }^{36}$ T. K. Gaisser, ${ }^{39}$ J. Gallagher, ${ }^{34}$ E. Ganster, ${ }^{1}$ S. Garrappa ${ }^{55}$ L. Gerhardt, ${ }^{9}$ K. Ghorbani, ${ }^{35}$ T. Glauch, ${ }^{25}$ T. Glüsenkamp, ${ }^{24}$ A. Goldschmidt, ${ }^{9}$ J. G. Gonzalez, ${ }^{39}$ D. Grant, ${ }^{22}$ T. Grégoire, ${ }^{52}$ Z. Griffith, ${ }^{35}$ S. Griswold ${ }^{45}$ M. Günder, ${ }^{1}$ M. Gündüz,${ }^{11}$ C. Haack, ${ }^{1}$ A. Hallgren,${ }^{53}$ R. Halliday, ${ }^{22}$ L. Halve, ${ }^{1}$ F. Halzen,${ }^{35}$ K. Hanson, ${ }^{35}$ A. Haungs, ${ }^{29}$ D. Hebecker, ${ }^{10}$ D. Heereman,${ }^{12}$ P. Heix, ${ }^{1}$ K. Helbing, ${ }^{54}$ R. Hellauer, ${ }^{17}$ F. Henningsen, ${ }^{25}$ S. Hickford, ${ }^{54}$ J. Hignight, ${ }^{23}$ G. C. Hill, ${ }^{2}$ K. D. Hoffman, ${ }^{17}$ R. Hoffmann, ${ }^{54}$ T. Hoinka, ${ }^{21}$ B. Hokanson-Fasig, ${ }^{35}$ K. Hoshina, ${ }^{35,}$ F. Huang, ${ }^{52}$ M. Huber, ${ }^{25}$ T. Huber, ${ }^{29,55}$ K. Hultqvist,${ }^{46}$ M. Hünnefeld, ${ }^{21}$ R. Hussain, ${ }^{35}$ S. In, ${ }^{48}$ N. Iovine, ${ }^{12}$ A. Ishihara, ${ }^{15}$ M. Jansson, ${ }^{46}$ G. S. Japaridze,${ }^{5}$ M. Jeong, ${ }^{48}$ K. Jero, ${ }^{35}$ B. J. P. Jones, ${ }^{4}$ F. Jonske, ${ }^{1}$ R. Joppe, ${ }^{1}$ D. Kang, ${ }^{29}$ W. Kang,${ }^{48}$ A. Kappes,${ }^{38}$ D. Kappesser, ${ }^{36}$ T. Karg, ${ }^{55}$ M. Karl, ${ }^{25}$ A. Karle, ${ }^{35}$ U. Katz, ${ }^{24}$ M. Kauer, ${ }^{35}$ J. L. Kelley, ${ }^{35}$ A. Kheirandish, ${ }^{35}$ J. Kim, ${ }^{48}$ T. Kintscher, ${ }^{55}$ J. Kiryluk $\odot,{ }^{47}$ T. Kittler ${ }^{24}$ S. R. Klein, ${ }^{8,9}$ R. Koirala, ${ }^{39}$ H. Kolanoski, ${ }^{10}$ L. Köpke, ${ }^{36}$ C. Kopper, ${ }^{22}$ S. Kopper, ${ }^{50}$ D. J. Koskinen, ${ }^{20}$ M. Kowalski, ${ }^{10,55}$ K. Krings, ${ }^{25}$ G. Krückl, ${ }^{36}$ N. Kulacz, ${ }^{23}$ N. Kurahashi, ${ }^{42}$ A. Kyriacou, ${ }^{2}$ J. L. Lanfranchi, ${ }^{52}$ M. J. Larson, ${ }^{17}$ F. Lauber, ${ }^{54}$ J. P. Lazar, ${ }^{35}$ K. Leonard, ${ }^{35}$ M. Lesiak-Bzdak, ${ }^{47}$ A. Leszczyńska, ${ }^{29}$ M. Leuermann, ${ }^{1}$ Q. R. Liu, ${ }^{35}$ E. Lohfink, ${ }^{36}$ C. J. Lozano Mariscal, ${ }^{38}$ L. Lu, ${ }^{15}$ F. Lucarelli, ${ }^{26}$ J. Lünemann, ${ }^{13}$ W. Luszczak, ${ }^{35}$ Y. Lyu, ${ }^{8,9}$ W. Y. Ma ${ }^{55}$ J. Madsen, ${ }^{44}$ G. Maggi, ${ }^{13}$ K. B. M. Mahn, ${ }^{22}$ Y. Makino, ${ }^{15}$ P. Mallik, ${ }^{1}$ K. Mallot,${ }^{35}$ S. Mancina,${ }^{35}$ I. C. Mariş, ${ }^{12}$ R. Maruyama,${ }^{40}$ K. Mase,${ }^{15}$ R. Maunu,${ }^{17}$ F. McNally, ${ }^{33}$ K. Meagher, ${ }^{35}$ M. Medici, ${ }^{20}$ A. Medina, ${ }^{19}$ M. Meier, ${ }^{21}$ S. Meighen-Berger, ${ }^{25}$ G. Merino, ${ }^{35}$ T. Meures, ${ }^{12}$ J. Micallef, ${ }^{22}$

D. Mockler, ${ }^{12}$ G. Momenté, ${ }^{36}$ T. Montaruli, ${ }^{26}$ R. W. Moore, ${ }^{23}$ R. Morse,${ }^{35}$ M. Moulai, ${ }^{14}$ P. Muth, ${ }^{1}$ R. Nagai,${ }^{15}$

U. Naumann, ${ }^{54}$ G. Neer, ${ }^{22}$ H. Niederhausen, ${ }^{47,25}$ M. U. Nisa, ${ }^{22}$ S. C. Nowicki, ${ }^{22}$ D. R. Nygren, ${ }^{9}$ A. Obertacke Pollmann, ${ }^{54}$ M. Oehler, ${ }^{29}$ A. Olivas, ${ }^{17}$ A. O'Murchadha, ${ }^{12}$ E. O'Sullivan, ${ }^{46}$ T. Palczewski, ${ }^{8,9}$ H. Pandya, ${ }^{39}$ D. V. Pankova, ${ }^{52}$ N. Park,${ }^{35}$ P. Peiffer ${ }^{36}$ C. Pérez de los Heros,${ }^{53}$ S. Philippen, ${ }^{1}$ D. Pieloth, ${ }^{21}$ S. Pieper,${ }^{54}$ E. Pinat, ${ }^{12}$ A. Pizzuto,${ }^{35}$ M. Plum, ${ }^{37}$ A. Porcelli, ${ }^{27}$ P. B. Price, ${ }^{8}$ G. T. Przybylski, ${ }^{9}$ C. Raab, ${ }^{12}$ A. Raissi, ${ }^{16}$ M. Rameez,${ }^{20}$ L. Rauch, ${ }^{55}$ K. Rawlins, ${ }^{3}$ I. C. Rea,${ }^{25}$ A. Rehman, ${ }^{39}$ R. Reimann, ${ }^{1}$ B. Relethford, ${ }^{42}$ M. Renschler, ${ }^{29}$ G. Renzi, ${ }^{12}$ E. Resconi, ${ }^{25}$ W. Rhode, ${ }^{21}$ M. Richman, ${ }^{42}$ S. Robertson, ${ }^{9}$ M. Rongen, ${ }^{1}$ C. Rott, ${ }^{48}$ T. Ruhe, ${ }^{21}$ D. Ryckbosch,${ }^{27}$ D. Rysewyk, ${ }^{22}$ I. Safa, ${ }^{35}$ S. E. Sanchez Herrera, ${ }^{22}$ A. Sandrock, ${ }^{21}$ J. Sandroos, ${ }^{36}$ M. Santander, ${ }^{50}$ S. Sarkar, ${ }^{41}$ S. Sarkar, ${ }^{23}$ K. Satalecka, ${ }^{55}$ M. Schaufel, ${ }^{1}$ H. Schieler,${ }^{29}$ P. Schlunder, ${ }^{21}$ T. Schmidt, ${ }^{17}$ A. Schneider ${ }^{35}$ J. Schneider, ${ }^{24}$ F. G. Schröder, ${ }^{29,39}$ L. Schumacher, ${ }^{1}$ S. Sclafani, ${ }^{42}$ D. Seckel, ${ }^{39}$

S. Seunarine, ${ }^{44}$ S. Shefali, ${ }^{1}$ M. Silva, ${ }^{35}$ R. Snihur, ${ }^{35}$ J. Soedingrekso, ${ }^{21}$ D. Soldin,${ }^{39}$ M. Song, ${ }^{17}$ G. M. Spiczak,${ }^{44}$

C. Spiering, ${ }^{55}$ J. Stachurska, ${ }^{55}$ M. Stamatikos ${ }^{19}$ T. Stanev, ${ }^{39}$ R. Stein, ${ }^{55}$ J. Stettner, ${ }^{1}$ A. Steuer, ${ }^{36}$ T. Stezelberger, ${ }^{9}$

R. G. Stokstad, ${ }^{9}$ A. Stöß1, ${ }^{15}$ N. L. Strotjohann, ${ }^{55}$ T. Stürwald, ${ }^{1}$ T. Stuttard, ${ }^{20}$ G. W. Sullivan, ${ }^{17}$ I. Taboada, ${ }^{6}$ F. Tenholt, ${ }^{11}$

S. Ter-Antonyan, ${ }^{7}$ A. Terliuk, ${ }^{55}$ S. Tilav,${ }^{39}$ K. Tollefson, ${ }^{22}$ L. Tomankova, ${ }^{11}$ C. Tönnis, ${ }^{49}$ S. Toscano, ${ }^{12}$ D. Tosi, ${ }^{35}$

A. Trettin, ${ }^{55}$ M. Tselengidou, ${ }^{24}$ C. F. Tung, ${ }^{6}$ A. Turcati ${ }^{25}$ R. Turcotte, ${ }^{29}$ C. F. Turley, ${ }^{52}$ B. Ty, ${ }^{35}$ E. Unger, ${ }^{53}$

M. A. Unland Elorrieta, ${ }^{38}$ M. Usner, ${ }^{55}$ J. Vandenbroucke, ${ }^{35}$ W. Van Driessche, ${ }^{27}$ D. van Eijk, ${ }^{35}$ N. van Eijndhoven, ${ }^{13}$ J. van Santen, ${ }^{55}$ S. Verpoest, ${ }^{27}$ M. Vraeghe, ${ }^{27}$ C. Walck ${ }^{46}$ A. Wallace, ${ }^{2}$ M. Wallraff, ${ }^{1}$ N. Wandkowsky, ${ }^{35}$ T. B. Watson, ${ }^{4}$ C. Weaver, ${ }^{23}$ A. Weindl, ${ }^{29}$ M. J. Weiss, ${ }^{52}$ J. Weldert, ${ }^{36}$ C. Wendt, ${ }^{35}$ J. Werthebach, ${ }^{35}$ B. J. Whelan, ${ }^{2}$ N. Whitehorn, ${ }^{32}$ K. Wiebe, ${ }^{36}$ C. H. Wiebusch, ${ }^{1}$ L. Wille, ${ }^{35}$ D. R. Williams, ${ }^{50}$ L. Wills, ${ }^{42}$ M. Wolf, ${ }^{25}$ J. Wood ${ }^{35}$ T. R. Wood, ${ }^{23}$ K. Woschnagg, ${ }^{8}$ G. Wrede, ${ }^{24}$ D. L. Xu, ${ }^{35}$ X. W. Xu, ${ }^{7}$ Y. Xu, ${ }^{47}$ J. P. Yanez, ${ }^{23}$ G. Yodh, ${ }^{28}$ S. Yoshida, ${ }^{15}$ T. Yuan, ${ }^{35}$ and M. Zöcklein ${ }^{1}$

(IceCube Collaboration) 
${ }^{1}$ III. Physikalisches Institut, RWTH Aachen University, D-52056 Aachen, Germany

${ }^{2}$ Department of Physics, University of Adelaide, Adelaide, 5005, Australia

${ }^{3}$ Department of Physics and Astronomy, University of Alaska Anchorage,

3211 Providence Drive, Anchorage, Alaska 99508, USA

${ }^{4}$ Department of Physics, University of Texas at Arlington,

502 Yates Street, Science Hall Rm 108, Box 19059, Arlington, Texas 76019, USA

${ }^{5}$ CTSPS, Clark-Atlanta University, Atlanta, Georgia 30314, USA

${ }^{6}$ School of Physics and Center for Relativistic Astrophysics, Georgia Institute of Technology, Atlanta, Georgia 30332, USA

${ }^{7}$ Department of Physics, Southern University, Baton Rouge, Louisiana 70813, USA

${ }^{8}$ Department of Physics, University of California, Berkeley, California 94720, USA

${ }^{9}$ Lawrence Berkeley National Laboratory, Berkeley, California 94720, USA

${ }^{10}$ Institut für Physik, Humboldt-Universität zu Berlin, D-12489 Berlin, Germany

${ }^{11}$ Fakultät für Physik \& Astronomie, Ruhr-Universität Bochum, D-44780 Bochum, Germany

${ }^{12}$ Université Libre de Bruxelles, Science Faculty CP230, B-1050 Brussels, Belgium

${ }^{13}$ Vrije Universiteit Brussel (VUB), Dienst ELEM, B-1050 Brussels, Belgium

${ }^{14}$ Department of Physics, Massachusetts Institute of Technology, Cambridge, Massachusetts 02139, USA

${ }^{15}$ Department of Physics and Institute for Global Prominent Research, Chiba University, Chiba 263-8522, Japan

${ }^{16}$ Department of Physics and Astronomy, University of Canterbury, Private Bag 4800, Christchurch, New Zealand

${ }^{17}$ Department of Physics, University of Maryland, College Park, Maryland 20742, USA

${ }^{18}$ Department of Astronomy, Ohio State University, Columbus, Ohio 43210, USA

${ }^{19}$ Department of Physics and Center for Cosmology and Astro-Particle Physics, Ohio State University, Columbus, Ohio 43210, USA

${ }^{20}$ Niels Bohr Institute, University of Copenhagen, DK-2100 Copenhagen, Denmark

${ }^{21}$ Department of Physics, TU Dortmund University, D-44221 Dortmund, Germany

${ }^{22}$ Department of Physics and Astronomy, Michigan State University, East Lansing, Michigan 48824, USA

${ }^{23}$ Department of Physics, University of Alberta, Edmonton, Alberta, Canada T6G $2 E 1$

${ }^{24}$ Erlangen Centre for Astroparticle Physics, Friedrich-Alexander-Universität Erlangen-Nürnberg, D-91058 Erlangen, Germany

${ }^{25}$ Physik-department, Technische Universität München, D-85748 Garching, Germany

${ }^{26}$ Département de Physique Nucléaire et Corpusculaire, Université de Genève, CH-1211 Genève, Switzerland

${ }^{27}$ Department of Physics and Astronomy, University of Gent, B-9000 Gent, Belgium

${ }^{28}$ Department of Physics and Astronomy, University of California, Irvine, California 92697, USA

${ }^{29}$ Karlsruhe Institute of Technology, Institut für Kernphysik, D-76021 Karlsruhe, Germany

${ }^{30}$ Department of Physics and Astronomy, University of Kansas, Lawrence, Kansas 66045, USA

${ }^{31}$ SNOLAB, 1039 Regional Road 24, Creighton Mine 9, Lively, Ontario, Canada P3Y IN2

${ }^{32}$ Department of Physics and Astronomy, UCLA, Los Angeles, California 90095, USA

${ }^{33}$ Department of Physics, Mercer University, Macon, Georgia 31207-0001, USA

${ }^{34}$ Department of Astronomy, University of Wisconsin, Madison, Wisconsin 53706, USA

${ }^{35}$ Department of Physics and Wisconsin IceCube Particle Astrophysics Center, University of Wisconsin, Madison, Wisconsin 53706, USA

${ }^{36}$ Institute of Physics, University of Mainz, Staudinger Weg 7, D-55099 Mainz, Germany

${ }^{37}$ Department of Physics, Marquette University, Milwaukee, Wisconsin, 53201, USA

${ }^{38}$ Institut für Kernphysik, Westfälische Wilhelms-Universität Münster, D-48149 Münster, Germany

${ }^{39}$ Bartol Research Institute and Department of Physics and Astronomy, University of Delaware, Newark, Delaware 19716, USA

${ }^{40}$ Department of Physics, Yale University, New Haven, Connecticut 06520, USA

${ }^{41}$ Department of Physics, University of Oxford, Parks Road, Oxford OX1 3PU, United Kingdom

${ }^{42}$ Department of Physics, Drexel University, 3141 Chestnut Street, Philadelphia, Pennsylvania 19104, USA

${ }^{43}$ Physics Department, South Dakota School of Mines and Technology, Rapid City, South Dakota 57701, USA

${ }^{44}$ Department of Physics, University of Wisconsin, River Falls, Wisconsin 54022, USA

${ }^{45}$ Department of Physics and Astronomy, University of Rochester, Rochester, New York 14627, USA

${ }^{46}$ Oskar Klein Centre and Department of Physics, Stockholm University, SE-10691 Stockholm, Sweden

${ }^{47}$ Department of Physics and Astronomy, Stony Brook University, Stony Brook, New York 11794-3800, USA

${ }^{48}$ Department of Physics, Sungkyunkwan University, Suwon 16419, Korea

${ }^{49}$ Institute of Basic Science, Sungkyunkwan University, Suwon 16419, Korea

${ }^{50}$ Department of Physics and Astronomy, University of Alabama, Tuscaloosa, Alabama 35487, USA

${ }^{51}$ Department of Astronomy and Astrophysics, Pennsylvania State University, University Park, Pennsylvania 16802, USA

${ }^{52}$ Department of Physics, Pennsylvania State University, University Park, Pennsylvania 16802, USA 
${ }^{53}$ Department of Physics and Astronomy, Uppsala University, Box 516, S-75120 Uppsala, Sweden

${ }^{54}$ Department of Physics, University of Wuppertal, D-42119 Wuppertal, Germany

${ }^{55}$ DESY, D-15738 Zeuthen, Germany

(Received 26 January 2020; revised 2 June 2020; accepted 20 July 2020; published 17 September 2020)

\begin{abstract}
We report on the first measurement of the astrophysical neutrino flux using particle showers (cascades) in IceCube data from 2010-2015. Assuming standard oscillations, the astrophysical neutrinos in this dedicated cascade sample are dominated ( $\sim 90 \%)$ by electron and tau flavors. The flux, observed in the sensitive energy range from $16 \mathrm{TeV}$ to $2.6 \mathrm{PeV}$, is consistent with a single power-law model as expected from Fermi-type acceleration of high energy particles at astrophysical sources. We find the flux spectral index to be $\gamma=$ $2.53 \pm 0.07$ and a flux normalization for each neutrino flavor of $\phi_{\text {astro }}=1.66_{-0.27}^{+0.25}$ at $E_{0}=100 \mathrm{TeV}$, in agreement with IceCube's complementary muon neutrino results and with all-neutrino flavor fit results. In the measured energy range we reject spectral indices $\gamma \leq 2.28$ at $\geq 3 \sigma$ significance level. Because of high neutrino energy resolution and low atmospheric neutrino backgrounds, this analysis provides the most detailed characterization of the neutrino flux at energies below $\sim 100 \mathrm{TeV}$ compared to previous IceCube results. Results from fits assuming more complex neutrino flux models suggest a flux softening at high energies and a flux hardening at low energies ( $p$ value $\geq 0.06$ ). The sizable and smooth flux measured below $\sim 100 \mathrm{TeV}$ remains a puzzle. In order to not violate the isotropic diffuse gamma-ray background as measured by the Fermi Large Area Telescope, it suggests the existence of astrophysical neutrino sources characterized by dense environments which are opaque to gamma rays.
\end{abstract}

DOI: 10.1103/PhysRevLett.125.121104

In 2013 IceCube discovered a diffuse and isotropic flux of neutrinos of astrophysical origin [1-3]. In 2018, an active galactic nucleus (AGN) with a relativistic jet pointing towards the Earth, the blazar TXS $0506+056$, was identified as the first possible extragalactic source of astrophysical neutrinos and cosmic ray accelerator [4,5]. In diffuse neutrino flux measurements one aims to gain insights into astrophysical neutrino production mechanisms, typically associated with cosmic ray acceleration at the source, and interactions either with surrounding gas $(p p)$ or photons $(p \gamma)$. The Fermi shock acceleration mechanism of high energy cosmic rays, in sources such as an AGN [6-11], predicts the flux of neutrinos to follow a single power law $E^{-\gamma}$ with a baseline spectral index of $\gamma \sim 2$ for strong shocks $[12,13]$. The spectral index and flux normalization factors carry information about neutrino sources and the environment [14,15]. Different production mechanisms together with energy losses of pions and muons lead, depending on energy, to different neutrino flavor compositions at sources and, after neutrino oscillations over astrophysical distances, at the Earth [16-24]. The main goal of astrophysical neutrino flux measurements is a characterization of its energy dependence in a flavor dependent way and in a wide energy range [25-32], relevant for ultrahigh energy cosmic rays and QCD physics. Since the diffuse Galactic emission component, based on models of galactic particle propagation and interactions [33], is subdominant [34,35], of particular interest is the energy range $\sim 10-100 \mathrm{TeV}$. In this energy region, hardly accessible to muon neutrinos, several source models, including AGN cores [36,37], predict a sizable energy dependent flux. In this Letter we present the first results on the astrophysical flux of electron and tau neutrinos determined with six years of IceCube data.

IceCube is a neutrino observatory comprising 5160 digital optical modules (DOMs) [38] distributed over one cubic kilometer in the Antarctic ice. Charged particles, which are produced in neutrino interactions, emit Cherenkov light while propagating through the ice. The Cherenkov light detected by the optical sensors forms three types of patterns, muon tracks (starting inside or going through the detector) and cascades. Single cascades are electromagnetic and/or hadronic particle showers produced by (i) electron or low energy tau neutrinos scattering inelastically off target nucleons through a $W$ boson, (ii) neutrinos of all flavors scattering inelastically off target nucleons through a $Z$ boson, or (iii) electron antineutrinos interacting with atomic electrons to form a $W^{-}$boson, the Glashow resonance [39]. Although the angular resolution of cascades is limited $\left(>8^{\circ}\right)$ [35], their energy resolution $(\sim 15 \%)$ [40] as well as their low atmospheric neutrino background make the cascade channel particularly well suited for measuring and characterizing the energy dependent astrophysical neutrino flux [41].

We analyzed six years of IceCube cascade data, collected in 2010-2015. We used IceCube Monte Carlo simulation packages to simulate the cosmic ray background with CORSIKA [42] and single muons from cosmic rays withMuonGun [43]. For the cosmic ray primary flux we used the Gaisser-H3a [44] model and SIBYLL 2.1 [45] as the hadronic interaction model. High energy neutrino interactions were generated with the NuGen 
software package based on Ref. [46]. The total $\nu N$ deep inelastic scattering cross section is from Ref. [47]. Astrophysical neutrino event selection efficiencies were tested assuming as baseline an $E^{-2}$ flux with equal numbers of neutrinos and antineutrinos, and with an equal neutrino flavor mixture at Earth: $\left(\nu_{e}: \nu_{\mu}: \nu_{\tau}\right)_{E}=$ $\left(\bar{\nu}_{e}: \bar{\nu}_{\mu}: \bar{\nu}_{\tau}\right)_{E}=0.5: 0.5: 0.5$. The conventional atmospheric neutrino flux from pion and kaon decays was modeled according to Ref. [48], with primary cosmic ray flux modifications according to the Gaisser-H3a model [44]. It is in agreement, in the energy range relevant to this analysis $E>400 \mathrm{GeV}$, with the atmospheric neutrino flux measurements by Super-Kamiokande [49], AMANDA-II [50,51], IceCube [52-54], and ANTARES [55]. Atmospheric neutrinos originating from the decays of charm or heavier mesons produced in air showers, so-called prompt neutrinos, are yet to be detected. We used the BERSS model [56] to predict the contribution from prompt neutrinos to the total neutrino flux, and the atmospheric neutrino self-veto effect calculations from Ref. [57], tuned to match our full CORSIKA Monte Carlo simulations.

The analyzed data consist of two sets: 2010-2011 (two years, sample $A$ ) [58] and 2012-2015 (four years, sample $B$ ) [59-61]. Events from both samples passed IceCube's dedicated online cascade filter, which utilizes results of simple muon and cascade reconstruction algorithms. The cascade filter reduces the cosmic ray background rate from $\sim 2.7 \mathrm{kHz}$ to $\sim 30 \mathrm{~Hz}$, while retaining $\sim 90 \%$ of the expected astrophysical neutrinos and $\sim 70 \%$ of the conventional atmospheric neutrinos. In order to further reduce backgrounds and ensure high neutrino induced cascade signal efficiencies and good cascade energy resolution, a fiducial volume selection on the reconstructed cascade vertex position was imposed. A straight cut selection method was used to select signal cascades in sample $A(E>10 \mathrm{TeV})$ [58] and in the high energy $(E>60 \mathrm{TeV})$ subset of sample $B[59,61]$. It builds on methods developed in previous IceCube searches dedicated to astrophysical cascades performed with partial detector configurations during IceCube construction periods [62-64]. A significant improvement was achieved by applying a boosted decision tree [65] method in the low energy $(\sim 400 \mathrm{GeV}<E<60 \mathrm{TeV})$ subset of sample $B$ to classify events according to their topology into muon track background, signal neutrino induced cascades, and muon starting track events $[59,60]$. The obtained cascade sample has low (8\%) muon background contamination. Lowering the energy threshold from $10 \mathrm{TeV}$ (sample $A$ ) to $\sim 400 \mathrm{GeV}$ (sample $B$ ) substantially reduces systematic uncertainties in this measurement. Reconstructed cascade energy distributions for sample $A$ and for sample $B$ after all selections are shown as black points in Fig. 1. About $60 \%$ of the cascades identified in this analysis and with reconstructed energies above $60 \mathrm{TeV}$ do not contribute to
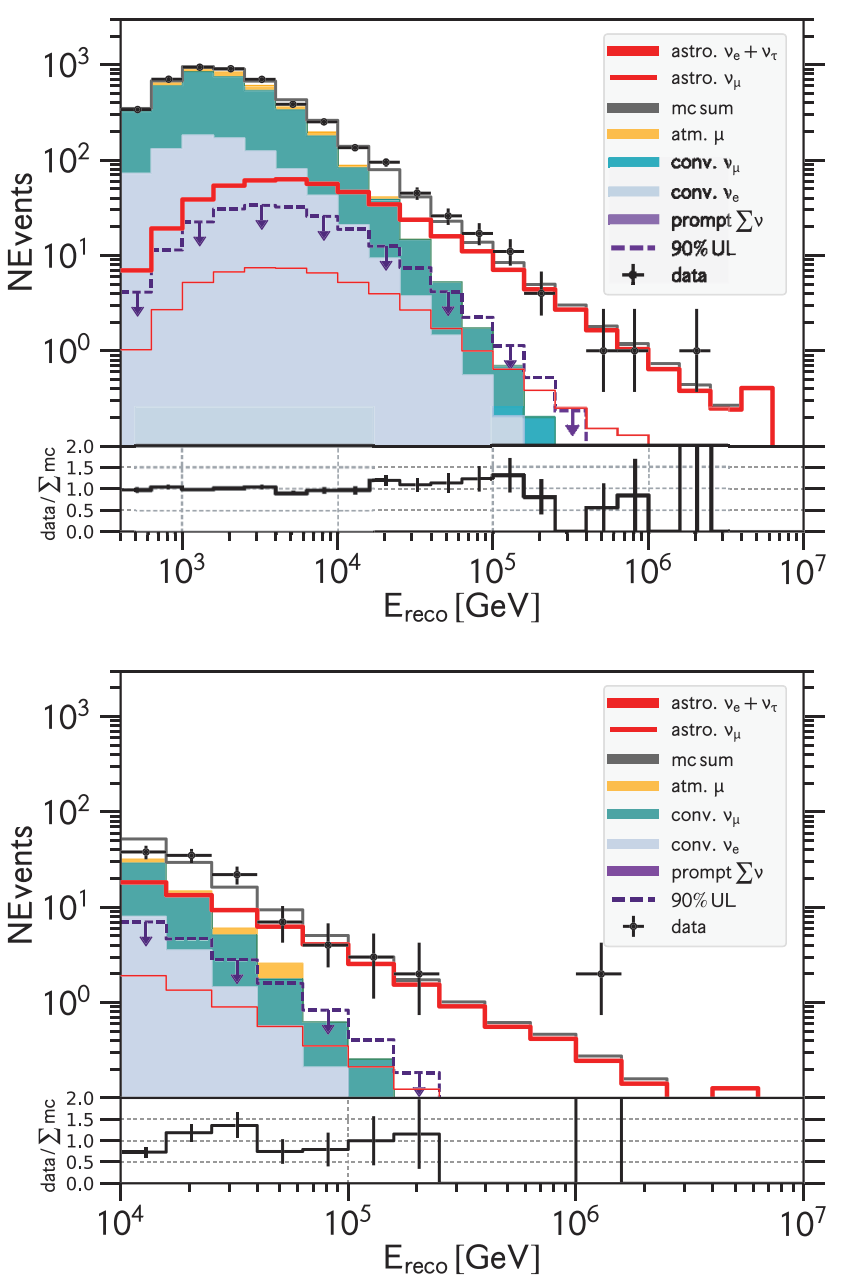

FIG. 1. Reconstructed cascade energy distribution. Black points are data, with statistical uncertainties, acquired during the observation period. Continuous lines are Monte Carlo simulations as labeled in the legend. The atmospheric background histograms are stacked (filled colors). Shown are best fit distributions assuming a single power-law model of the astrophysical neutrino flux (Table II). Top: data from 2012-2015 (sample B). Bottom: data from 2010-2011 (sample A).

the high energy starting events (HESEs) [28] cascade data sample for the same period (2010-2015). Monte Carlo simulations show that at $10 \mathrm{TeV}$ this analysis increases the total expected number of electron neutrinos by a factor of 10 compared to the medium energy starting events (MESEs) analysis [29].

We determined the astrophysical neutrino flux, characterized by parameters $\boldsymbol{\theta}_{\boldsymbol{r}}$, by maximizing a binned Poisson likelihood $L\left(\boldsymbol{\theta}_{\boldsymbol{r}}, \boldsymbol{\theta}_{\boldsymbol{s}} \mid \mathbf{n}\right)$. The $\boldsymbol{\theta}_{\boldsymbol{s}}$ are the nuisance parameters, and $\mathbf{n}=\left(n_{1}, \ldots, n_{m}\right)$ is the vector of observed event counts $n_{i}$ in the $i$ th bin. The fit was performed in bins of three observables: event type (cascade, muon track, muon starting track), reconstructed energy, and reconstructed zenith angle in the range $0-\pi$, as shown in Table I. In this analysis, the log-likelihood function is defined, up to a constant, as 
TABLE I. The binning of observables (reconstructed energy and zenith) used in the maximum likelihood fit. Energy ranges are given in logarithmic units, $\log _{10} E / \mathrm{GeV}$, and zenith ranges are given in radians. The three bins' ranges in $\cos ($ Zenith) are $(-1,0.2,0.6,1)$

\begin{tabular}{lcccc}
\hline \hline $\begin{array}{l}\text { Sample \& event } \\
\text { type }\end{array}$ & $\begin{array}{c}\text { Energy } \\
\text { NBins }\end{array}$ & $\begin{array}{c}\text { Energy } \\
\text { range }\end{array}$ & $\begin{array}{c}\text { Zenith } \\
\text { NBins }\end{array}$ & $\begin{array}{c}\text { Zenith } \\
\text { range }\end{array}$ \\
\hline$A$ cascade & 15 & $4.0-7.0$ & 3 & $0-\pi$ \\
$B$ cascade & 22 & $2.6-7.0$ & 3 & $0-\pi$ \\
$B \mu$ starting track & 11 & $2.6-4.8$ & 1 & $0-\pi$ \\
$B \mu$ track & 1 & $2.6-4.8$ & 1 & $0-\pi$ \\
\hline \hline
\end{tabular}

$$
\begin{aligned}
\log L\left(\boldsymbol{\theta}_{\boldsymbol{r}}, \boldsymbol{\theta}_{\boldsymbol{s}} \mid \mathbf{n}\right)=\sum_{i=1}^{m}\left[n_{i} \log \mu_{i}\left(\boldsymbol{\theta}_{\boldsymbol{r}}, \boldsymbol{\theta}_{\boldsymbol{s}}\right)-\mu_{i}\left(\boldsymbol{\theta}_{\boldsymbol{r}}, \boldsymbol{\theta}_{\boldsymbol{s}}\right)\right] \\
+\frac{1}{2}\left[\left(\frac{\epsilon_{\mathrm{eff}}^{\mathrm{DOM}}-\hat{\epsilon}_{\mathrm{eff}}^{\mathrm{DOM}}}{\sigma_{\epsilon}^{\mathrm{DOM}}}\right)^{2}+\left(\frac{\epsilon_{\mathrm{abs}}^{\mathrm{HI}}-\hat{\epsilon}_{\mathrm{abs}}^{\mathrm{HI}}}{\sigma_{\epsilon}^{\mathrm{HI}}}\right)^{2}\right. \\
\left.+\left(\frac{\Delta \gamma_{\mathrm{CR}}-\hat{\Delta} \gamma_{\mathrm{CR}}}{\sigma_{\Delta \gamma_{\mathrm{CR}}}}\right)^{2}\right] \\
+\frac{1}{2}\left(\boldsymbol{\epsilon}^{\mathrm{BI}}-\hat{\boldsymbol{\epsilon}}^{\mathrm{BI}}\right)^{T} \boldsymbol{\Sigma}_{\mathrm{BI}}^{-1}\left(\boldsymbol{\epsilon}^{\mathrm{BI}}-\hat{\boldsymbol{\epsilon}}^{\mathrm{BI}}\right)
\end{aligned}
$$

The expected, from Monte Carlo simulations, number of events in the $i$ th bin is defined as $\mu_{i}=\mu_{i}^{\text {atm. } \mu}+\mu_{i}^{\text {conv. } \nu}+$ $\mu_{i}^{\text {prompt } \nu}+\mu_{i}^{\text {astro. } \nu}$, the sum of background cosmic ray muons, conventional and prompt atmospheric neutrinos, and astrophysical neutrinos. The nuisance parameters $\boldsymbol{\theta}_{\boldsymbol{s}}$ contribute additive penalty terms to the log-likelihood function, Eq. (1). They account for detector related systematic uncertainties, comprised of the DOM optical efficiency, $\epsilon_{\mathrm{eff}}^{\mathrm{DOM}}$, optical properties (scattering and absorption length) of the bulk ice (BI), $\epsilon_{\text {scat }}^{\mathrm{BI}}$ and $\epsilon_{\mathrm{abs}}^{\mathrm{BI}}$, and of the refrozen drilled hole ice $(\mathrm{HI}), \epsilon_{\text {scat }}^{\mathrm{HI}}$. The bivariate covariance matrix $\boldsymbol{\Sigma}_{\mathrm{BI}}$ takes into account correlations between the two components of $\mathbf{\epsilon}^{\mathrm{BI}}=\left(\epsilon_{\mathrm{scat}}^{\mathrm{BI}}, \epsilon_{\mathrm{abs}}^{\mathrm{BI}}\right)$. Other systematic uncertainties are due to uncertainties on the

TABLE II. Best fit values and uncertainties for all parameters included in the single power-law fit.

\begin{tabular}{lcc}
\hline \hline Parameter & Prior constraint & Result $\pm 1 \sigma(<90 \%$ upper limit $)$ \\
\hline$\gamma$ & $\ldots$ & $\mathbf{2 . 5 3} \pm \mathbf{0 . 0 7}$ \\
$\phi_{\text {astro }}$ & $\ldots$ & $\mathbf{1 . 6 6 _ { - 0 . 2 7 } ^ { + 0 . 2 5 }}$ \\
$\phi_{\text {conv }}$ & $\ldots$ & $\left(1.07_{-0.12}^{+0.13}\right) \times \Phi_{\text {HKKMS06 }}$ \\
$\phi_{\text {prompt }}$ & $\ldots$ & $<5.0 \times \Phi_{\text {BERSS }}$ \\
$\phi_{\text {muon }}$ & $\ldots$ & $1.45 \pm 0.04$ \\
$\Delta \gamma_{\mathrm{CR}}$ & $0.00 \pm 0.05$ & $0.02 \pm 0.03$ \\
$\epsilon_{\text {scat }}^{\mathrm{BI}}$ & $1.00 \pm 0.07$ & $1.02 \pm 0.03$ \\
$\epsilon_{\text {abs }}^{\mathrm{BI}}$ & $1.00 \pm 0.07$ & $1.03_{-0.04}^{+0.05}$ \\
$\epsilon_{\text {scat }}^{\mathrm{HI}}$ & $\cdots$ & $1.72 \pm 0.19$ \\
$\epsilon_{\mathrm{eff}}^{\mathrm{DOM}}$ & $0.99 \pm 0.10$ & $1.03_{-0.07}^{+0.08}$ \\
\hline \hline
\end{tabular}

TABLE III. Number of events for the six years cascade data. The number of astrophysical neutrinos results from the single power-law best fit. Numbers of events given in brackets refer to neutrinos with reconstructed energies above $10 \mathrm{TeV}$. The number of atmospheric tau neutrinos is negligible. Number of Glashow resonance (astro. GR) events are evaluated assuming $p p$-type sources in the 4-8 PeV energy range.

\begin{tabular}{lccc}
\hline \hline Number of events & $\nu_{e}+\bar{\nu}_{e}$ & $\nu_{\mu}+\bar{\nu}_{\mu}$ & $\nu_{\tau}+\bar{\nu}_{\tau}$ \\
\hline astro. & $303_{-45}^{+46}$ & $59_{-7}^{+8}$ & $204_{-27}^{+28}$ \\
& $\left(127_{-12}^{+12}\right)$ & $\left(22_{-2}^{+2}\right)$ & $\left(80_{-7}^{+7}\right)$ \\
astro. GR & $0.73_{-0.22}^{+0.31}$ & $\ldots$ & $\ldots$ \\
atmo. conv. & $851_{-23}^{+23}$ & $2901_{-65}^{+64}$ & $\ldots$ \\
& $\left(50_{-3}^{+3}\right)$ & $\left(143_{-8}^{+8}\right)$ & $\ldots$ \\
atmo. prompt & $<192$ & $<32$ & $\ldots$ \\
& $(<57)$ & $(<7)$ & $\ldots$ \\
\hline \hline
\end{tabular}

cosmic ray flux index $\Delta \gamma_{\mathrm{CR}}$, on the flux normalizations of the cosmic ray muon $\phi_{\text {muon }}$, atmospheric conventional $\phi_{\text {conv }}$ and prompt $\phi_{\text {prompt }}$ neutrino backgrounds. Uncertainties in the atmospheric neutrino flux prediction related to hadronic interaction models [66-69] have been studied using the MCEq [70] package. They were found small and thus neglected.

We performed several fits considering different functional forms of the astrophysical neutrino flux. All models assume equal numbers of neutrinos and anti-

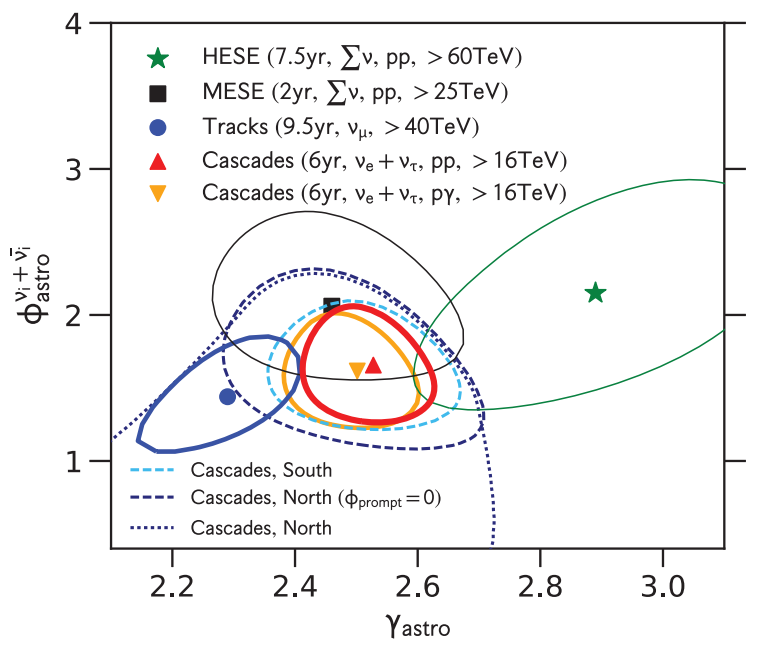

FIG. 2. $68 \%$ C.L. profile likelihood contours for the single power-law astrophysical neutrino flux fit parameters, the flux normalization (per neutrino flavor), and the spectral index. Shown are results for the combined 2010-2015 (six years) cascade analysis. Red (yellow) curves are obtained assuming a $p p(p \gamma)$ neutrino production mechanism at the source, respectively. Other IceCube results are shown as blue, green, and gray curves for $\nu_{\mu}$ [26] and for all-neutrino flavor HESE [28] and MESE [29] analyses. 
neutrinos and equal neutrino flavors at Earth. First we describe the results obtained for the single power-law flux model:

$$
\Phi_{\text {astro }}^{\nu+\bar{v}}(E) / C_{0}=\phi_{\text {astro }} \times\left(E / E_{0}\right)^{-\gamma}
$$

where $\quad C_{0}=3 \times 10^{-18} \mathrm{GeV}^{-1} \cdot \mathrm{cm}^{-2} \cdot \mathrm{s}^{-1} \cdot \mathrm{sr}^{-1}$ and $E_{0}=100 \mathrm{TeV}$. We find the following best fit parameters: the flux spectral index $\gamma=2.53 \pm 0.07$ and the flux normalization for each neutrino flavor $\phi_{\text {astro }}=$ $1.66_{-0.27}^{+0.25}$ at $E_{0}=100 \mathrm{TeV}$. The result for the measured electron and tau neutrino flux $\Phi_{\text {astro }}^{\nu_{e}+\bar{\nu}_{e}}+\Phi_{\text {astro }}^{\nu_{\tau}+\bar{\nu}_{\tau}}$ changes insignificantly, if we include variations in the injected flavor ratio at astrophysical sources $\left(\nu_{e}: \nu_{\mu}: \nu_{\tau}\right)_{S}=$ $\left(1-f_{\mu}^{S}: f_{\mu}^{S}: 0\right)$ through an additional nuisance parameter $0 \leq f_{\mu}^{S} \leq 1$, as shown in the Supplemental Material Fig. 1 (right) [71]. The sensitive energy range, defined as the smallest range where a nonzero astrophysical flux is consistent with the data at $90 \%$ C.L. [60], ranges from $16 \mathrm{TeV}$ to $2.6 \mathrm{PeV}$. The best fit values of all physics and nuisance fit parameters and their uncertainties are given in Table II. Figure 1 shows the reconstructed cascade energy distributions for data and for Monte Carlo simulations with the signal and background contributions scaled according to the best fit values of all fit parameters. The agreement between data and simulations is very good with a goodness-of-fit [72] $p$ value of 0.88 [60]. The number of neutrino events based on the best fit results are shown in Table III. The contribution from astrophysical electron and tau neutrinos to the cascade samples strongly dominates over the small (12\%) contribution from astrophysical muon neutrinos. The energy

TABLE IV. $\quad C_{0}=3 \times 10^{-18} \mathrm{GeV}^{-1} \cdot \mathrm{cm}^{-2} \cdot \mathrm{s}^{-1} \cdot \mathrm{sr}^{-1}$ and $E_{0}=100 \mathrm{TeV}$. Fit results for different hypotheses, assuming the baseline $\left(\nu_{e}: \nu_{\mu}: \nu_{\tau}\right)_{E}=\left(\bar{\nu}_{e}: \bar{\nu}_{\mu}: \bar{\nu}_{\tau}\right)_{E}=0.5: 0.5: 0.5$ flavor composition expected for $p p$ sources (hypotheses $\left.A-F\right)$ and $\left(\nu_{e}: \nu_{\mu}: \nu_{\tau}\right)_{E}=$ 0.78:0.61:0.61, $\left(\bar{\nu}_{e}: \bar{\nu}_{\mu}: \bar{\nu}_{\tau}\right)_{E}=0.22: 0.39: 0.39$ expected for $p \gamma$ sources (hypothesis $G$ ). Goodness of fit test used in this work is the saturated Poisson likelihood test [60,72]. The corresponding goodness of fit $p$ values have been calculated as described in Ref. [60] (Section 5.5). Significance $\sigma$ of alternative, more complex astrophysical flux models over single power-law model as determined from toy experiments. The significance of the single-power law fit with respect to the background only hypothesis $\left(\Phi_{\text {astro }}=0\right)$ is $9.9 \sigma$. All significances are given using the one-sided convention.

\begin{tabular}{|c|c|c|c|c|c|}
\hline Hypothesis & Flux model $\left(\nu_{\text {astro }}\right)$ & $\Phi_{\text {astro }}^{\nu+\bar{\nu}}(E, \cos \theta) / C_{0}=$ & Result & g.o.f $s$ & icance $[\sigma]$ \\
\hline$A$ & Single power law & $\Phi_{0}\left(E / E_{0}\right)^{-\gamma}$ & $\begin{array}{c}\gamma=2.53_{-0.07}^{+0.07} \\
\Phi_{0}=1.66_{-0.27}^{+0.25}\end{array}$ & 0.88 & $\cdots$ \\
\hline$B$ & Single power law with cutoff & $\Phi_{0}\left(E / E_{0}\right)^{-\gamma} \exp \left(-E / E_{\text {cut }}\right)$ & $\begin{array}{c}\gamma=2.45_{-0.11}^{+0.09} \\
\Phi_{0}=1.83_{-0.31}^{+0.37} \\
\log _{10}\left(E_{\text {cut }} / \mathrm{GeV}\right)=6.4_{-0.4}^{+0.9}\end{array}$ & 0.79 & 1.0 \\
\hline$C$ & Log parabolic power law & $\begin{array}{l}\Phi_{0}\left(E / E_{0}\right)^{-\Gamma(E)} \\
\Gamma(E)=\gamma+b \log \left(E / E_{0}\right)\end{array}$ & $\begin{array}{c}\gamma=2.58_{-0.10}^{+0.10} \\
\Phi_{0}=1.81_{-0.29}^{+0.31}\end{array}$ & 0.79 & 1.6 \\
\hline$D$ & Broken power law & 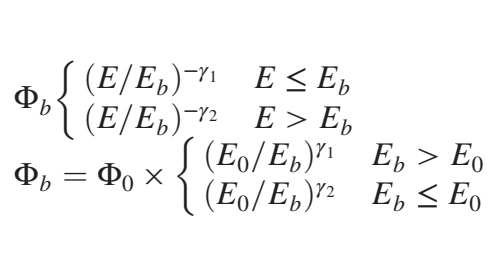 & $\begin{array}{c}b=0.07_{-0.05}^{+0.05} \\
\Phi_{0}=1.71_{-0.29}^{+0.65} \\
\log _{10}\left(E_{b} / \mathrm{GeV}\right)=4.6_{-0.2}^{+0.5} \\
\gamma_{1}=2.11_{-0.67}^{+0.29} \\
\gamma_{2}=2.75_{-0.14}^{+0.29}\end{array}$ & 0.82 & 1.3 \\
\hline E & $\begin{array}{l}\text { Single power lawwith cutoff } \\
+\sum \text { BL Lac [Padovani BL Lac] }\end{array}$ & $\begin{array}{l}\Phi_{0}\left(E / E_{0}\right)^{-\gamma} \exp \left(-E / E_{\text {cut }}\right)+ \\
\quad Y_{\nu \gamma} \times f(E)\end{array}$ & $\begin{array}{c}\gamma=2.0_{-0.4}^{+0.3} \\
\Phi_{0}=4.3_{-1.6}^{+3.2} \\
\log _{10}\left(E_{\text {cut }} / \mathrm{GeV}\right)=5.1_{-0.2}^{+0.3}\end{array}$ & 0.78 & 1.1 \\
\hline$F$ & Two hemispheres & $\begin{cases}\Phi_{N}\left(E / E_{0}\right)^{-\gamma_{N}} & \cos \theta \leq 0 \\
\Phi_{S}\left(E / E_{0}\right)^{-\gamma_{S}} & \cos \theta>0\end{cases}$ & $\begin{array}{c}Y_{\nu \gamma}=0.20_{-0.09}^{+0.12} \\
\gamma_{N}=2.45_{-0.36}^{+0.17} \\
\Phi_{N}=1.3_{-1.0}^{+0.7} \\
\gamma_{S}=2.52_{-0.11}^{+0.10}\end{array}$ & 0.87 & 0.0 \\
\hline$G$ & Single power law $(p \gamma)$ & $\Phi_{0}\left(E / E_{0}\right)^{-\gamma}$ & $\begin{aligned} \Phi_{S} & =1.62_{-0.29}^{+0.30} \\
\gamma & =2.50_{-0.07}^{+0.07} \\
\Phi_{0} & =1.62_{-0.27}^{+0.25}\end{aligned}$ & 0.88 & 0.7 \\
\hline
\end{tabular}


and zenith angle dependence of the measured flux is consistent with expectations for a flux of neutrinos of astrophysical origin. The 68\% C.L. profile likelihood contours for the correlated spectral index and flux normalization are shown in Fig. 2 as a red curve. Similar results (yellow curve, $\gamma=2.50 \pm 0.07$ and $\phi_{\text {astro }}=1.62_{-0.27}^{+0.25}$ ) were obtained under the assumption that the astrophysical neutrino flux originated from the $p \gamma$-type source where we used the at-earth flavor ratios, $\left(\nu_{e}: \nu_{\mu}: \nu_{\tau}\right)_{E}=0.78: 0.61: 0.61$ and $\left(\bar{\nu}_{e}: \bar{\nu}_{\mu}: \bar{\nu}_{\tau}\right)_{E}=$ $0.22: 0.39: 0.39$ [73], and assumed the single power-law flux. No significant difference has been observed between the fluxes from the northern and southern skies (dashed cyan and blue lines in Fig. 2). Since the atmospheric self-veto effect $[43,57,74,75]$ reduces atmospheric neutrino background in the southern sky, the astrophysical flux is measured more precisely in the southern than in the northern hemisphere, $\gamma_{S}=2.52_{-0.11}^{+0.10}$ and $\gamma_{N}=2.45_{-0.36}^{+0.17}$ (Table IV, hypothesis $F$ ). Other IceCube results are shown as blue, green, and black curves for the muon neutrinos [26], HESEs [28] and MESEs (Medium Energy Starting Events, $E>25 \mathrm{TeV}$ ) [29] analyses. Only the muon neutrino sample is uncorrelated with cascade events from this analysis. The muon neutrino flux, measured for energies above $40 \mathrm{TeV}$ from the northern sky, is in agreement with the cascade result at the level of $1.5 \sigma$ corresponding to a $p$ value of 0.07 . The electron and tau neutrino (cascade) and all-neutrino flavor (HESE and MESE) measurements, which are correlated, are consistenin the overlapping energy range.

The results from fits beyond a single power-law model assumption are described below. In the differential model we assumed the flux follows an $E^{-2}$ spectrum in the individual neutrino energy segments with independent normalizations [60]. The corresponding fit results, which indicate the strength of the astrophysical neutrino flux, are shown as black points in Fig. 3. The fit results assuming other hypotheses are shown as curves with functional forms given in Table IV. The red curve is the result of the single power-law fit (hypothesis $A$ ) with the band indicating allowed parameters at $68 \%$ C.L.. Single power-law fit results, obtained in the southern and northern skies separately (hypothesis $F$ ) lead to similar results. Other models assume additional features in the flux shape, such as a cutoff (hypotheses $B$ and $E$ ), break in the spectrum (hypothesis $D$ ), energy dependence of the spectral index (hypothesis $C$ ) as well as an additional neutrino emission component at high neutrino energies from the population of Blazar Lacertae blazars (hypothesis $E$ ). The latter has been modeled according to Ref. [76] with one free parameter, the neutrino to $\gamma$-ray intensity ratio, $Y_{\nu \gamma}$. The fit results are given in Table IV. Although not statistically significant, the results (hypotheses $C, D$, and $E$ ) indicate an overall soft spectral index $(\gamma \sim 2.4-2.6)$, a softening of

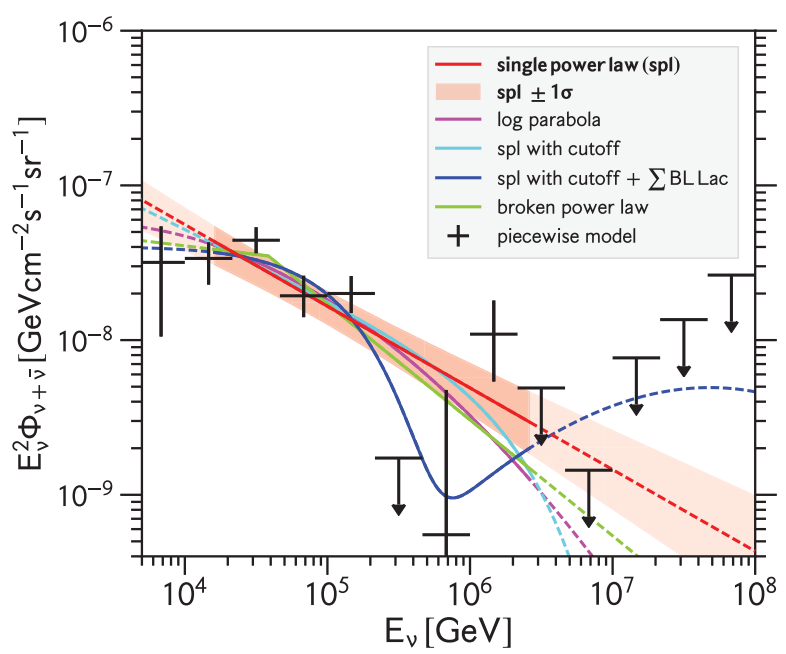

FIG. 3. Astrophysical neutrino flux per neutrino flavor as a function of energy. Black crosses represent the differential flux model best fit results for the 2010-2015 (six years) cascade data. Colored solid (dashed) curves represent astrophysical neutrino flux models in (outside of) the sensitive energy range from $16 \mathrm{TeV}$ to $2.6 \mathrm{PeV}$. Their functional forms as well as fit results are given in Table IV. The $1 \sigma$ data uncertainties, data limits, and uncertainty band correspond to the $68 \%$ C.L. simultaneous coverage for the unbroken single power-law flux.

spectral index with energy from $\gamma \sim 2.0$ to $\gamma \sim 2.75$ above $\sim 40 \mathrm{TeV}$, or a cutoff in the flux from the low energy component at energies as low as $\sim 0.1 \mathrm{PeV}$. The nonzero contribution from the BL Lac neutrino flux component (hypothesis E), which is proportional to the $Y_{\nu \gamma}$, is statistically nonsignificant. We thus placed an upper limit on the ratio $Y_{\nu, \gamma}<0.41$ at $90 \%$ C.L., leading to the conclusion that a significant fraction of the $\gamma$-ray emission from BL Lacs is due to leptonic processes, in agreement with the IceCube limit at ultrahigh energies [77,78]. Current statistics are not sufficient to distinguish between models that go beyond the single power law (hypotheses $B-F$, Table IV). The most significant extension to the single power law is hypothesis $C$, assuming energy dependent spectral indices, with a $p$ value of 0.06 .

In summary, our results are consistent with the hypothesis that the flux of astrophysical electron and tau neutrinos follows a single power law, with a spectral index of $\gamma=2.53 \pm 0.07$ and a flux normalization for each neutrino flavor of $\phi_{\text {astro }}=\left(1.66_{-0.27}^{+0.25}\right)$ at $E_{0}=100 \mathrm{TeV}$. In the measured energy range we reject spectral indices $\gamma \leq 2.28$ at $\geq 3 \sigma$ level. The sizable and smooth flux measured below $\sim 100 \mathrm{TeV}$ remains a puzzle. In order to not violate the isotropic diffuse gamma-ray background [79], it suggests the existence of astrophysical neutrino sources characterized by dense environments which are opaque to gamma rays $[80,81]$.

The IceCube collaboration acknowledges the significant contributions to this Letter from the Stony Brook 
University. We acknowledge the support from the following agencies: USA-U.S. National Science FoundationOffice of Polar Programs, U.S. National Science Foundation-Physics Division, Wisconsin Alumni Research Foundation, Center for High Throughput Computing (CHTC) at the University of WisconsinMadison, Open Science Grid (OSG), Extreme Science and Engineering Discovery Environment (XSEDE), U.S. Department of Energy-National Energy Research Scientific Computing Center, Particle Astrophysics Research Computing Center at the University of Maryland, Institute for Cyber-Enabled Research at Michigan State University, and Astroparticle physics computational facility at Marquette University; Belgium-Funds for Scientific Research (FRS-FNRS and FWO), FWO Odysseus and Big Science programmes, and Belgian Federal Science Policy Office (Belspo); Germany-Bundesministerium für Bildung und Forschung (BMBF), Deutsche Forschungsgemeinschaft (DFG), Helmholtz Alliance for Astroparticle Physics (HAP), Initiative and Networking Fund of the Helmholtz Association, Deutsches Elektronen Synchrotron (DESY), and High Performance Computing cluster of the RWTH Aachen; Sweden-Swedish Research Council, Swedish Polar Research Secretariat, Swedish National Infrastructure for Computing (SNIC), and Knut and Alice Wallenberg Foundation; Australia-Australian Research Council; Canada-Natural Sciences and Engineering Research Council of Canada, Calcul Québec, Compute Ontario, Canada Foundation for Innovation, WestGrid, and Compute Canada; DenmarkVillum Fonden, Danish National Research Foundation (DNRF), Carlsberg Foundation; New Zealand-Marsden Fund; Japan-Japan Society for Promotion of Science (JSPS) and Institute for Global Prominent Research (IGPR) of Chiba University; Korea-National Research Foundation of Korea (NRF); Switzerland-Swiss National Science Foundation (SNSF); United KingdomDepartment of Physics, University of Oxford.

*analysis@icecube.wisc.edu

†Also at Università di Padova, I-35131 Padova, Italy.

${ }^{*}$ Also at National Research Nuclear University, Moscow Engineering Physics Institute (MEPhI), Moscow 115409, Russia.

${ }^{\S}$ Also at Earthquake Research Institute, University of Tokyo, Bunkyo, Tokyo 113-0032, Japan.

[1] M. G. Aartsen et al. (IceCube Collaboration), First Observation of PeV-Energy Neutrinos with IceCube, Phys. Rev. Lett. 111, 021103 (2013).

[2] M. G. Aartsen et al. (IceCube Collaboration), Evidence for high-energy extraterrestrial neutrinos at the icecube detector, Science 342, 1242856 (2013).

[3] M. G. Aartsen et al. (IceCube Collaboration), Observation of High-Energy Astrophysical Neutrinos in Three Years of IceCube Data, Phys. Rev. Lett. 113, 101101 (2014).
[4] M. G. Aartsen et al. (IceCube, Fermi-LAT, MAGIC, AGILE, ASAS-SN, HAWC, H.E.S.S., and INTEGRAL Collaborations), Multimessenger observations of a flaring blazar coincident with high-energy neutrino IceCube170922A, Science 361 (2018).

[5] M. G. Aartsen et al. (IceCube Collaboration), Neutrino emission from the direction of the blazar TXS 0506+056 prior to the IceCube-170922A alert, Science 361, 147 (2018).

[6] R. J. Protheroe and D. Kazanas, On the origin of relativistic particles and gamma-rays in quasars, Astrophys. J. 265, 620 (1983).

[7] D. Kazanas and D. Ellison, The central engine of quasars and active galactic nuclei: hadronic interactions of shockaccelerated relativistic protons, Astrophys. J. 304, 178 (1986).

[8] M. Sikora et al., Electron injection by relativistic protons in active galactic nuclei, Astrophys. J. Lett. 320, L81 (1987).

[9] F. W. Stecker, C. Done, M. H. Salamon, and P. Sommers, High-Energy Neutrinos from Active Galactic Nuclei, Phys. Rev. Lett. 66, 2697 (1991).

[10] F. W. Stecker, C. Done, M. H. Salamon, and P. Sommers, Erratum:High-Energy Neutrinos from Active Galactic Nuclei, Phys. Rev. Lett. 69, 2738(E) (1992).

[11] K. Mannheim and P. L. Biermann, Photomeson production in active galactic nuclei, Astron. Astrophys. 221, 211 (1989).

[12] A. R. Bell, The acceleration of cosmic rays in shock fronts, Mon. Not. R. Astron. Soc. 182, 147 (1978).

[13] T. K. Gaisser, Cosmic Rays and Particle Physics (Cambridge University Press, Cambridge, England, 1990).

[14] W. Winter, Photohadronic origin of the TeV-PeV neutrinos observed in IceCube, Phys. Rev. D 88, 083007 (2013).

[15] K. Murase, M. Ahlers, and B.C. Lacki, Testing the hadronuclear origin of $\mathrm{PeV}$ neutrinos observed with IceCube, Phys. Rev. D 88, 121301(R) (2013).

[16] J. G. Learned and S. Pakvasa, Detecting $\nu_{\tau}$ oscillations at PeV energies, Astropart. Phys. 3, 267 (1995).

[17] H. Athar, C. S. Kim, and J. Lee, Intrinsic and oscillated astrophysical neutrino flavor ratios revisited, Mod. Phys. Lett. A 21, 1049 (2006).

[18] T. Kashti and E. Waxman, Astrophysical Neutrinos: Flavor Ratios Depend on Energy, Phys. Rev. Lett. 95, 181101 (2005).

[19] S. R. Klein, R. E. Mikkelsen, and J. Becker Tjus, Muon acceleration in cosmic-ray sources, Astrophys. J. 779, 106 (2013).

[20] P. Lipari, M. Lusignoli, and D. Meloni, Flavor composition and energy spectrum of astrophysical neutrinos, Phys. Rev. D 75, 123005 (2007).

[21] M. Bustamante, J. F. Beacom, and W. Winter, Theoretically Palatable Flavor Combinations of Astrophysical Neutrinos, Phys. Rev. Lett. 115, 161302 (2015).

[22] A. Esmaili and Y. Farzan, An analysis of cosmic neutrinos: Flavor composition at source and neutrino mixing parameters, Nucl. Phys. B821, 197 (2009).

[23] I. Esteban, M.C. Gonzalez-Garcia, A. HernandezCabezudo, M. Maltoni, and T. Schwetz, Global analysis of three-flavour neutrino oscillations: synergies and tensions 
in the determination of $\hat{\mathrm{I}}_{3} 23, \hat{\mathrm{I}}^{\prime} \mathrm{CP}$, and the mass ordering, J. High Energy Phys. 01 (2019) 106.

[24] NuFIT 4.1, www.nu-fit.org (2019).

[25] M. G. Aartsen et al. (IceCube Collaboration), Observation and Characterization of a Cosmic Muon Neutrino Flux from the Northern Hemisphere using six years of IceCube data, Astrophys. J. 833, 3 (2016).

[26] M. G. Aartsen et al. (IceCube Collaboration), Measurement of the diffuse astrophysical muon-neutrino spectrum with ten years of IceCube data, Proc. Sci., ICRC2019 (2019) 1017.

[27] M. G. Aartsen et al. (IceCube Collaboration), Measurements using the inelasticity distribution of multi-TeV neutrino interactions in IceCube, Phys. Rev. D 99, 032004 (2019).

[28] M. G. Aartsen et al. (IceCube Collaboration), Characterization of the astrophysical diffuse neutrino flux with IceCube high-energy starting events, Proc. Sci., ICRC2019 (2019) 1004.

[29] M. G. Aartsen et al. (IceCube Collaboration), Atmospheric and astrophysical neutrinos above $1 \mathrm{TeV}$ interacting in IceCube, Phys. Rev. D 91, 022001 (2015).

[30] M. G. Aartsen et al. (IceCube Collaboration), A combined maximum-likelihood analysis of the high-energy astrophysical neutrino flux measured with IceCube, Astrophys. J. 809, 98 (2015).

[31] A. Albert et al. (Antares Collaboration), All-flavor search for a diffuse flux of cosmic neutrinos with nine years of ANTARES data, Astrophys. J. Lett. 853, L7 (2018).

[32] A. D. Avrorin et al. (Baikal-GVD Collaboration), Search for cascade events with Baikal-GVD, Proc. Sci., ICRC2019 (2019) 0873.

[33] D. Gaggero, D. Grasso, A. Marinelli, A. Urbano, and M. Valli, THE gamma-ray and neutrino sky: A consistent picture of Fermi-lat, Milagro, and IceCube results, Astrophys. J. Lett. 815, L25 (2015).

[34] A. Albert et al. (Antares and IceCube Collaborations), Joint constraints on galactic diffuse neutrino emission from the ANTARES and IceCube neutrino telescopes, Astrophys. J. 868, L20 (2018).

[35] M. G. Aartsen et al. (IceCube Collaboration), Search for sources of astrophysical neutrinos using seven years of IceCube cascade events, Astrophys. J. 886, 12 (2019).

[36] Y. Inoue, S. Khangulyan, D. Inoue, and A. Doi, On high-energy particles in accretion disk coronae of supermassive black holes: Implications for MeV gammarays and high-energy neutrinos from AGN cores, Astrophys. J. 880, 40 (2019).

[37] K. Murase, S. S. Kimura, and P. Meszaros, Hidden Cores of Active Galactic Nuclei as the Origin of Medium-Energy Neutrinos: Critical Tests with the MeV Gamma-Ray Connection, Phys. Rev. Lett. 125, 011101 (2020).

[38] M. G. Aartsen et al. (IceCube Collaboration), The IceCube neutrino observatory: Instrumentation and online systems, J. Instrum. 12, P03012 (2017).

[39] S. L. Glashow, Resonant scattering of antineutrinos, Phys. Rev. 118, 316 (1960).

[40] M. G. Aartsen et al. (IceCube Collaboration), Energy reconstruction methods in the IceCube neutrino telescope, J. Instrum. 9, P03009 (2014).
[41] J. F. Beacom and J. Candia, Shower power: Isolating the prompt atmospheric neutrino flux using electron neutrinos, J. Cosmol. Astropart. Phys. 11 (2004) 009.

[42] D. Heck et al., Technical Report No. FZKA 6019, Institute for Nuclear Physics, Karlsruhe Institute of Technology (KIT), 1998.

[43] J. van Santen, Ph.D. thesis, University of WisconsinMadison, 2014.

[44] T. K. Gaisser, Spectrum of cosmic-ray nucleons, kaon production, and the atmospheric muon charge ratio, Astropart. Phys. 35, 801 (2012).

[45] E. J. Ahn, R. Engel, T. K. Gaisser, P. Lipari, and T. Stanev, Cosmic ray interaction event generator SIBYLL 2.1, Phys. Rev. D 80, 094003 (2009).

[46] A. Gazizov and M.P. Kowalski, ANIS: High energy neutrino generator for neutrino telescopes, Comput. Phys. Commun. 172 (2005)

[47] A. Cooper-Sarkar, P. Mertsch, and S. Sarkar, The high energy neutrino cross-section in the Standard Model and its uncertainty, J. High Energy Phys. 08 (2011) 042.

[48] M. Honda, T. Kajita, K. Kasahara, S. Midorikawa, and T. Sanuki, Calculation of atmospheric neutrino flux using the interaction model calibrated with atmospheric muon data, Phys. Rev. D 75, 043006 (2007).

[49] E. Richard et al. (Super-K Collaboration), Measurements of the atmospheric neutrino flux by Super-Kamiokande: Energy spectra, geomagnetic effects, and solar modulation, Phys. Rev. D 94, 052001 (2016).

[50] R. Abbasi et al. (IceCube Collaboration), Determination of the atmospheric neutrino flux and searches for new physics with AMANDA-II, Phys. Rev. D 79, 102005 (2009).

[51] R. Abbasi, Y. Abdou, T. Abu-Zayyad, J. Adams, J.A. Aguilar, M. Ahlers, K. Andeen, J. Auffenberg, X. Bai, and M. Baker (IceCube Collaboration), The energy spectrum of atmospheric neutrinos between 2 and $200 \mathrm{TeV}$ with the AMANDA-II detector, Astropart. Phys. 34, 48 (2010).

[52] R. Abbasi et al. (IceCube Collaboration), Measurement of the atmospheric neutrino energy spectrum from $100 \mathrm{GeV}$ to $400 \mathrm{TeV}$ with IceCube, Phys. Rev. D 83, 012001 (2011).

[53] M. G. Aartsen et al. (IceCube Collaboration), Measurement of the atmospheric ve flux in IceCube, Phys. Rev. Lett. 110, 151105 (2013).

[54] M. G. Aartsen et al. (IceCube Collaboration), Measurement of the Atmospheric ve Spectrum with IceCube, Phys. Rev. D 91, 122004 (2015).

[55] S. Adrian-Martinez et al. (ANTARES Collaboration), Measurement of the atmospheric $\nu_{\mu}$ energy spectrum from $100 \mathrm{GeV}$ to $200 \mathrm{TeV}$ with the ANTARES telescope, Eur. Phys. J. C 73, 2606 (2013).

[56] A. Bhattacharya, R. Enberg, M. H. Reno, I. Sarcevic, and A. Stasto, Perturbative charm production and the prompt atmospheric neutrino flux in light of RHIC and LHC, J. High Energy Phys. 06 (2015) 110.

[57] T. K. Gaisser, K. Jero, A. Karle, and J. van Santen, Generalized self-veto probability for atmospheric neutrinos, Phys. Rev. D 90, 023009 (2014).

[58] M. G. Aartsen et al. (IceCube Collaboration), High energy astrophysical neutrino flux characteristics for neutrinoinduced cascades using IC79 and IC86-string IceCube conf, Proc. Sci., ICRC2015 (2015) 1109. 
[59] M. G. Aartsen et al. (IceCube Collaboration), High Energy Astrophysical Neutrino Flux Measurement Using Neutrinoinduced Cascades Observed in 4 Years of IceCube Data, Proc. Sci., ICRC2017 (2017) 968.

[60] H. Niederhausen, Ph.D. thesis, Stony Brook University, 2018.

[61] Y. Xu, Ph.D. Thesis, Stony Brook University, 2019.

[62] R. Abbasi et al. (IceCube Collaboration), First search for atmospheric and extraterrestrial neutrino-induced cascades with the IceCube detector, Phys. Rev. D 84, 072001 (2011).

[63] M. G. Aartsen et al. (IceCube Collaboration), Search for neutrino-induced particle showers with IceCube-40, Phys. Rev. D 89, 102001 (2014).

[64] M. G. Aartsen et al. (IceCube Collaboration), Search for diffuse astrophysical neutrinos with cascade events in the IceCube-59 detector, Proc. Sci., ICRC2013 (2013) 662, https://arxiv.org/pdf/1309.7003.pdf.

[65] T. Chen and C. Guestrin, XGBoost: A Scalable Tree Boosting System, Proceedings of the 22nd KDD (Association for Computing Machinery, New York, 2016), p. 785.

[66] F. Riehn et al., A new version of the event generator Sibyll, Proc. Sci., ICRC2015 (2015) 558.

[67] T. Pierog, I. Karpenko, J. M. Katzy, E. Yatsenko, and K. Werner, EPOS LHC: Test of collective hadronization with data measured at the CERN Large Hadron Collider, Phys. Rev. C 92, 034906 (2015).

[68] S. Roesler, R. Engel, and J. Ranft, The Monte Carlo Event Generator DPMJET-III, Advanced Monte Carlo for Radiation Physics, Particle Transport Simulation and Applications (Springer, Berlin, Heidelberg, 2001).

[69] A. Fedynitch and R. Engel, Revision of the high energy hadronic interaction models PHOJET/DPMJETIII, Proceedings of 14th International Conference on Nuclear Reaction Mechanisms (CERN, Geneva, 2015).

[70] A. Fedynitch et al., Calculation of conventional and prompt lepton fluxes at very high energy, EPJ Web Conf. 99, 08001 (2015).

[71] See Supplemental Material at http://link.aps.org/ supplemental/10.1103/PhysRevLett.125.121104 for the astrophysical neutrino flux parameters profile likelihood contours with different assumptions on the neutrino flavor composition.

[72] S. Baker and R. D. Cousins, Clarification of the use of chi square and likelihood functions in fits to histograms, Nucl. Instrum. Methods 221, 437 (1984).

[73] A. Bhattacharya, R. Gandhi, W. Rodejohann, and A. Watanabe, The Glashow resonance at IceCube: signatures, event rates and pp vs. $p \gamma$ interactions, J. Cosmol. Astropart. Phys. 10 (2011) 017.

[74] S. Schonert, T. K. Gaisser, E. Resconi, and O. Schulz, Vetoing atmospheric neutrinos in a high energy neutrino telescope, Phys. Rev. D 79, 043009 (2009).

[75] C. A. Argüelles, S. Palomares-Ruiz, A. Schneider, L. Wille, and T. Yuan, Unified atmospheric neutrino passing fractions for large-scale neutrino telescopes, J. Cosmol. Astropart. Phys. 07 (2018) 047.

[76] P. Padovani, M. Petropoulou, P. Giommi, and E. Resconi, A simplified view of blazars: the neutrino background, Mon. Not. R. Astron. Soc. 452, 1877 (2015).

[77] M. G. Aartsen et al. (IceCube Collaboration), Constraints on Ultrahigh-Energy Cosmic-Ray Sources from a Search for Neutrinos above $10 \mathrm{PeV}$ with IceCube, Phys. Rev. Lett. 117, 241101 (2016).

[78] M. G. Aartsen et al. (IceCube Collaboration), Erratum: Constraints on Ultrahigh-Energy Cosmic-Ray Sources from a Search for Neutrinos Above $10 \mathrm{PeV}$ with IceCube, Phys. Rev. Lett. 119, 259902(E) (2017).

[79] M. Ackermann et al. (Fermi-LAT Collaboration), The spectrum of isotropic diffuse gamma-ray emission between $100 \mathrm{MeV}$ and $820 \mathrm{GeV}$, Astrophys. J. 799, 86 (2015).

[80] K. Murase, D. Guetta, and M. Ahlers, Hidden Cosmic-Ray Accelerators as an Origin of TeV-PeV Cosmic Neutrinos, Phys. Rev. Lett. 116, 071101 (2016).

[81] K. Bechtol, M. Ahlers, M. Di Mauro, M. Ajello, and J. Vandenbroucke, Evidence against star-forming galaxies as the dominant source of IceCube neutrinos, Astrophys. J. 836, 47 (2017). 